\title{
Diagnostic and prognostic value of microRNA-193b in patients with glioma and its effect on tumor progression
}

\author{
MINGTAO ZHU ${ }^{1 *}$, WEI ZHAO ${ }^{2 *}, \mathrm{HUI} \mathrm{ZHAO}^{3 *}$ and $\mathrm{JING}^{*} \mathrm{ZHANG}^{2}$ \\ ${ }^{1}$ Department of Neurosurgery, Xinglin Branch of The First Affiliated Hospital of Xiamen University, \\ Xiamen, Fujian 361003; ${ }^{2}$ Department of Neurosurgery, Heze Municipal Hospital, Heze, Shandong 274031; \\ ${ }^{3}$ Department of Pharmacology, Heze Medical College, Heze, Shandong 274000, P.R. China
}

Received November 22, 2018; Accepted August 8, 2019

DOI: $10.3892 / 01.2019 .10819$

\begin{abstract}
Emerging evidence has indicated the important roles of microRNAs (miRs) in the pathogenesis of cancer in humans. The present study sought to assess the expression patterns of miR-193b in patients with glioma, and investigated its clinical significance and biological function in this disease. The expression of miR-193b in the serum, tissues and cells of patients with glioma was analyzed using reverse transcription-quantitative PCR. Its diagnostic value was evaluated using the receiver operating characteristic (ROC) curve analysis, and its prognostic value was analyzed using Kaplan-Meier survival and Cox regression analyses. Experiments on glioma cells were conducted to explore the influence of miR-193b on proliferation, migration and invasion. Increased expression of miR-193b was observed in serum, tissues and cells of patients with glioma compared with the corresponding controls (all $\mathrm{P}<0.05)$. miR-193b expression was associated with the World Health Organization grading and the Karnofsky Performance Scale of the patients (all $\mathrm{P}<0.05$ ). The area under the curve of the ROC analysis of miR-193b was 0.903 , indicating its high diagnostic accuracy for glioma. High expression of miR-193b was associated with poor overall survival rate in patients $(\mathrm{P}=0.002)$. Therefore, miR-193b is a potential independent prognostic factor in glioma. Furthermore, the overexpression of this $\mathrm{miR}$ in glioma cells led to increased proliferation, migration and invasion, whereas its inhibition resulted in the opposite effects on these cell behaviors (all $\mathrm{P}<0.05$ ). Thus, the findings from the present study indicate that the overexpression of miR-193b serves as a useful biomarker for the diagnosis and prediction of prognosis in glioma. The upregulation of
\end{abstract}

Correspondence to: Professor Jing Zhang, Department of Neurosurgery, Heze Municipal Hospital, 2888 Caozhou Road, Heze, Shandong 274031, P.R. China

E-mail: chenguo8517685@163.com

*Contributed equally

Key words: microRNA-193b, diagnosis, prognosis, tumor progression, glioma
miR-193b expression may enhance glioma progression, and may therefore be a potential target for glioma therapy.

\section{Introduction}

Malignancies of the central nervous system represent one of the most serious health burdens worldwide, accounting for $1.9 \%$ of newly diagnosed cancer cases and $2.3 \%$ of cancer-associated deaths worldwide (1). Glioma, the most prevalent of the malignancies of the central nervous system, has been extensively studied due to its poor outcome and the lack of effective treatment (2). It is clinicopathologically classified into four grades, including pilocytic astrocytoma (grade I), diffuse astrocytoma (grade II), anaplastic astrocytoma (grade III), and glioblastoma (GBM; grade IV), according to the World Health Organization (WHO) grading criteria (3). Among these grades, GBM (WHO grade IV) is considered the most common type and is characterized by high invasion rates $(4,5)$. The significant biological heterogeneity of glioma hinders the understanding of the molecular mechanisms in tumor pathogenesis $(6,7)$. Currently, the 5-year survival rate of patients with glioma has improved as a result of the advances in therapeutic strategies, such as surgical procedures, radiotherapy and chemotherapy. However, the prognosis of patients with advanced stage glioma remains poor $(8,9)$. Therefore, the development of novel approaches is required to improve early diagnosis, prognosis and treatment of patients with glioma.

Accumulating evidence highlights the important roles of microRNAs (miRs/miRNAs) in the pathogenesis of multiple types of human cancer (10). Therefore, miRNAs have attracted increasing attention due to their significant diagnostic and prognostic values in patients with various types of cancer $(11,12)$. These small non-coding RNAs play pivotal roles in biological processes by regulating the expression of key genes at the post-transcriptional level (13). It is reported that miRNAs are stable in serum and can be extracted from clinical specimens easily, thus showing potential as diagnostic tools in the clinic (14). In addition, the abnormally expressed miRNAs in tumors are involved in tumor progression, through downregulation of oncogenes or tumor suppressors, and thus act as therapeutic targets in cancer treatment $(15,16)$. Thus, the clinical significance of novel aberrant miRNAs in the treatment of glioma was investigated. miR-193b has been 
investigated in several types of human carcinoma, such as liver, gastric and colorectal carcinoma (17-19). This miR was demonstrated to enhance cell proliferation and suppress cell apoptosis in liver cancer cells (17). In gastric cancer, overexpression of miR-193b in tumor cells resulted in the inhibition of cell proliferation, migration and invasion (18). In colorectal cancer, the aberrant expression of miR-193b in tumor tissues was determined as a prognostic biomarker for predicting overall survival in patients (19). A study by Zhong et al (20) demonstrated that miR-193b in glioma cells led to increased cell proliferation. However, the understanding of the clinical significance of miR-193b and its functional role in the progression of glioma remains limited.

To investigate the role of miR-193b in glioma further, the present study sought to assess the expression of miR-193b in glioma serum, tissues and cells, evaluate its clinical significance in diagnosis and prognosis, and explore its effects on glioma cell function.

\section{Materials and methods}

Patients and specimens collection. A total of 122 patients who were histologically diagnosed with glioma in Heze Municipal Hospital between June 2008 and May 2012 were recruited for the present study. The inclusion criteria for the glioma patients were as follows: i) Pathologically diagnosed as glioma; ii) had no preoperative therapy. The exclusion criteria were as follows: i) systemic infection; ii) diagnosed with other tumors. The present study also included 68 healthy volunteers, who received routine physical examination and had no history of malignancy. A volume of $5 \mathrm{ml}$ venous blood was obtained from the participants prior to surgical resection for the patients. The blood samples were placed in EDTA tubes and were used for serum isolation by centrifugation with $955.9 \times \mathrm{g}$ for $5 \mathrm{~min}$ at $4^{\circ} \mathrm{C}$. Glioma tissues and adjacent normal tissues, which were defined as $1 \mathrm{~cm}$ away from the lesions, were collected from the 122 patients during the surgery, and immediately frozen in liquid nitrogen for further use. Demographic and clinicopathological characteristics, including gender, age, tumor size, WHO grade (21) and Karnofsky Performance Scale (KPS) (22) were recorded and are listed in Table I. All patients were enrolled on a 5-year follow-up survey, and their survival data were obtained for the survival analysis. This study was approved by the Ethics Committee of Heze Municipal Hospital, and each participant provided written informed consent.

Cell culture and transfection. Glioma cell lines including T98G, A172 and LN229, and a normal human astrocyte cell line UC2 were purchased from the Type Culture Collection of the Chinese Academy of Sciences. Glioma U87 cell line of undetermined origin was obtained from the American Type Culture Collection (cat. no. ATCC ${ }^{\circledR} \mathrm{HTB}-14^{\mathrm{TM}}$ ). All cells were cultured in Dulbecco's modified Eagle's medium (DMEM), supplemented with 10\% FBS (both Gibco; Thermo Fisher Scientific, Inc.), in a humidified atmosphere at $5 \% \mathrm{CO}_{2}$ and $37^{\circ} \mathrm{C}$.

The expression of miR-193b in glioma cells was modulated by transfecting cells with $50 \mathrm{nM}$ miR-193b mimic (5'-AAC UGGCCCUCAAAGUCCCGCU-3'), 100 nM miR-193b inhibitor (5'-AGCGGGACUUUGAGGGCCAGUU-3') and $50 \mathrm{nM}$ corresponding negative controls mimic NC (5'-ACUACUGAG UGACAGUAGA-3') and $100 \mathrm{nM}$ inhibitor NC (5'-CAGUAC UUUUGUGUAGUACAA-3') (all from Shanghai GenePharma Co., Ltd.), using Lipofectamine ${ }^{\circledR} 2000$ (Invitrogen; Thermo Fisher Scientific, Inc.). The subsequent cell experiments were performed $48 \mathrm{~h}$ after the cell transfection.

RNA extraction and reverse transcription-quantitative $P C R$ $(R T-q P C R)$. Total RNA was extracted from glioma serum samples, tissues and cells using TRIzol reagent (Invitrogen; Thermo Fisher Scientific, Inc.). RT was conducted to synthesize cDNA from $1 \mu \mathrm{g}$ RNA, using a PrimeScript RT reagent kit (Takara Bio, Inc.) following the manufacturer's instructions with a following reaction condition: $42^{\circ} \mathrm{C}$ for $30 \mathrm{~min}, 85^{\circ} \mathrm{C}$ for 5 sec. The cDNA was subsequently used as the template for qPCR, which was carried out to evaluate the expression levels of miR-193b using a SYBR-Green I Master Mix kit (Invitrogen; Thermo Fisher Scientific, Inc.) and the 7300 Real-Time PCR System (Applied Biosystems; Thermo Fisher Scientific, Inc.). The reactions in this analysis used U6 as an internal control gene, and the thermocycling conditions were as follows: $95^{\circ} \mathrm{C}$ for $10 \mathrm{~min}, 95^{\circ} \mathrm{C}$ for $30 \mathrm{sec}, 60^{\circ} \mathrm{C}$ for $20 \mathrm{sec}, 72^{\circ} \mathrm{C}$ for $15 \mathrm{sec}$, for a total of 40 cycles. The oligonucleotide primer sequences were as follows: miR-193b forward, 3'-GCGCAACTGGCC CTCAAAG-5'; miR-193b reverse, 3'-CAGTGCAGGGTC CGAGGT-5'; U6 forward, 5'-GCTTCGGCAGCACATATA CTAAAAT-3'; and U6 reverse, 5'-CGCTTCACGAATTTG CGTGTCAT-3'. The final relative miR-193b expression was calculated using the $2^{-\Delta \Delta \mathrm{Cq}}$ method (23) and normalized to U6.

Cell proliferation analysis. Following cell transfection, the effect of miR-193b on the proliferation of glioma cell was examined using the Cell Counting Kit-8 (CCK-8; Dojindo Molecular Technologies, Inc.), as per the manufacturer's protocols. The stable transfected cells were seeded into 96-well plates with a cell density of $2 \times 10^{5}$ cells/well, and then maintained in a humidified incubator at $37^{\circ} \mathrm{C}$ for 3 days. At the culture times of $0,24,48$ and $72 \mathrm{~h}, \mathrm{CCK}-8$ reagent was added to the wells and incubated further for $2 \mathrm{~h}$. The cell viability was measured by reading the absorbance at a wavelength of $450 \mathrm{~nm}$, using a micro-plate analyzer (Bio-Rad Laboratories, Inc.).

Cell migration and invasion analysis. To explore the effect of miR-193b on cell migration and invasion, Transwell chambers with $8-\mu \mathrm{m}$ pore size (Corning, Inc.) were used. The chambers were coated with Matrigel for the invasion assay, and those without Matrigel were used for the migration assay. Stably transfected cells $\left(2 \times 10^{5}\right)$ were seeded in the upper chamber in serum-free DMEM. The lower chambers contained DMEM, with $10 \%$ FBS as the chemoattractant. After $48 \mathrm{~h}$ of incubation, the cells in the lower chamber were fixed with $4 \%$ paraformaldehyde for $10 \mathrm{~min}$ at room temperature and stained with $0.1 \%$ crystal violet for $20 \mathrm{~min}$ at room temperature. The cells were then counted using a light microscope (Olympus Corporation) at magnification, x200. All the experiments were performed in triplicate.

Western blot analysis. Proteins were extracted from the cells using RIPA lysis buffer (Thermo Fisher Scientific, Inc.), and 
Table I. Association between miR-193b expression level and clinicopathological features of patients with glioma.

\begin{tabular}{|c|c|c|c|c|c|c|c|}
\hline \multirow[b]{2}{*}{ Features } & \multirow[b]{2}{*}{ Patients, $\mathrm{n}(\mathrm{n}=122)$} & \multicolumn{2}{|c|}{ Serum miR-193b level } & \multirow[b]{2}{*}{ P-value } & \multicolumn{2}{|c|}{ Tissue miR-193b level } & \multirow[b]{2}{*}{ P-value } \\
\hline & & Low $(n=60)$ & High $(n=62)$ & & Low $(n=58)$ & $\operatorname{High}(\mathrm{n}=64)$ & \\
\hline Sex & & & & 0.483 & & & 0.601 \\
\hline Female & 45 & 24 & 21 & & 20 & 25 & \\
\hline Male & 77 & 36 & 41 & & 38 & 39 & \\
\hline Age, years & & & & 0.910 & & & 0.458 \\
\hline$\leq 50$ & 34 & 17 & 17 & & 18 & 16 & \\
\hline$>50$ & 88 & 43 & 45 & & 40 & 48 & \\
\hline Tumor size, $\mathrm{cm}$ & & & & 0.570 & & & 0.088 \\
\hline$\leq 3$ & 68 & 35 & 33 & & 37 & 31 & \\
\hline$>3$ & 54 & 25 & 29 & & 21 & 33 & \\
\hline WHO grade & & & & 0.030 & & & 0.004 \\
\hline I-II & 59 & 35 & 24 & & 36 & 23 & \\
\hline III-IV & 63 & 25 & 38 & & 22 & 41 & \\
\hline KPS & & & & 0.016 & & & 0.001 \\
\hline$\leq 90$ & 83 & 47 & 36 & & 48 & 35 & \\
\hline$>90$ & 39 & 13 & 26 & & 10 & 29 & \\
\hline
\end{tabular}

miR, microRNA; WHO, World Health Organization; KPS, Karnofsky Performance Scale.

the BCA method was used to examine the concentration of proteins. Proteins (20 $\mu \mathrm{g}$ per lane) were separated via SDS-PAGE (10\% gel) and transferred onto PVDF membranes (Merck KGaA). The membranes were incubated with primary antibodies against matrix metalloproteinase (MMP)-2 (1:1,000; cat. no. 4022S; Cell Signaling Technology, Inc.) and MMP-9 (1:1,000; cat. no. 2270S; Cell Signaling Technology, Inc.) overnight at $4^{\circ} \mathrm{C}$, after $4 \mathrm{~h}$ of blocking with $5 \%$ non-fat milk at room temperature. Horseradish peroxidase-labeled secondary antibodies (1:2,000; cat. no. A0192; Beyotime Institute of Biotechnology) were incubated at room temperature for $4 \mathrm{~h}$. $\beta$-actin was used as an internal control with an anti- $\beta$-actin (1:1,000; cat. no. AA128; Beyotime Institute of Biotechnology). The proteins were detected using an enhanced chemiluminescence reagent (Seven Sea Biotech), and were visualized using the Bio-Rad gel imaging system (Beijing Thmorgan Biotechnology, Inc.). The quantitative analysis for the proteins was performed using the IPP software (version 7.0; Media Cybernetics, Inc.).

Statistical analysis. Data in this study are presented as the mean \pm SD and were analyzed using SPSS version 18.0 (IBM Corp.) and GraphPad Prism version 5.0 software (GraphPad Software, Inc.). The differences between groups were assessed using Student's t-test and one-way ANOVA with Tukey's multiple comparison test. Association analysis between miR-193b and clinicopathological data was performed using the $\chi^{2}$ test. A receiver operating characteristic (ROC) curve was plotted to evaluate the diagnostic value of miR-193b, based on its serum expression levels. Kaplan-Meier survival and Cox regression analyses were adopted to examine the prognostic value of miR-193b. $\mathrm{P}<0.05$ was considered to indicate a statistically significant difference.

\section{Results}

Expression of miR-193b in glioma samples. To further understand the role of miR-193b in glioma, its expression in glioma samples and cells lines was quantified by RT-qPCR. As shown in Fig. 1A, the serum expression of miR-193b was significantly upregulated in patients with glioma compared with the healthy controls $(\mathrm{P}<0.01)$. Consistently, increased miR-193b expression was also observed in glioma tissues compared with the adjacent normal tissues $(\mathrm{P}<0.01$; Fig. $1 \mathrm{~B})$. To confirm these findings, the levels of miR-193b in glioma cell lines were also investigated, which demonstrated higher expression of miR-193b in four glioma cell lines than in the normal human astrocyte UC2 cells (all $\mathrm{P}<0.01$; Fig. 1C).

Association between miR-193b expression and clinicopathological features of patients with glioma. The present study subsequently explored the role of miR-193b in the development of glioma, by analyzing the association between miR-193b and the clinical data of patients. Firstly, the mean expression value of miR-193b (serum, 1.594; tissue, 0.084) was used as the cutoff values to classify the patients into low and high miR-193b expression groups. The findings from this analysis, summarized in Table I, revealed that the expression of miR-193b (in serum and tissues) was associated with WHO grade (serum, $\mathrm{P}=0.030$; tissue, $\mathrm{P}=0.004$ ) and KPS (serum, $\mathrm{P}=0.016$; tissue, $\mathrm{P}=0.001$ ) of patients. In contrast, no association was found between miR-193b expression and other parameters, such as gender, age and tumor size (all $\mathrm{P}>0.05)$.

Clinical significance of $m i R-193 b$ in the diagnosis and prognosis of glioma. The diagnostic and prognostic value of 
A

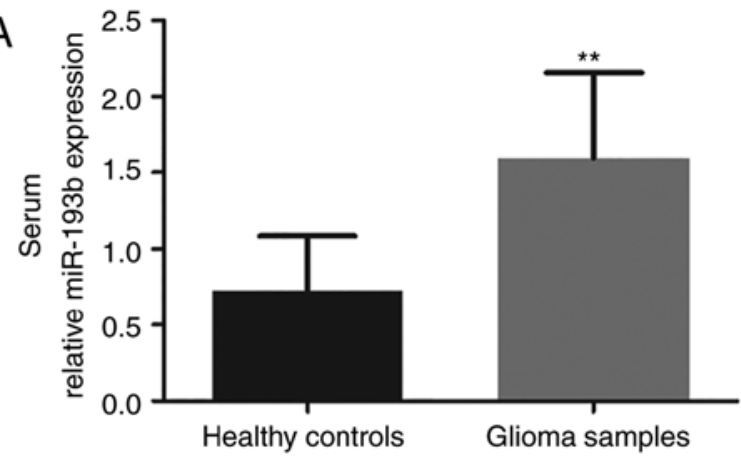

B

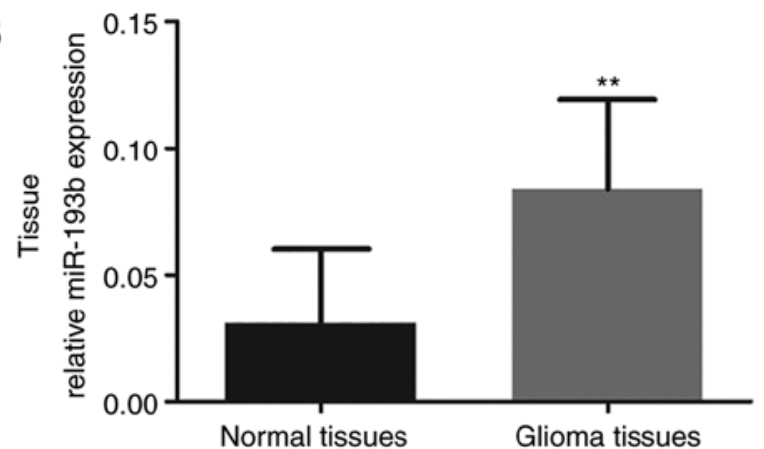

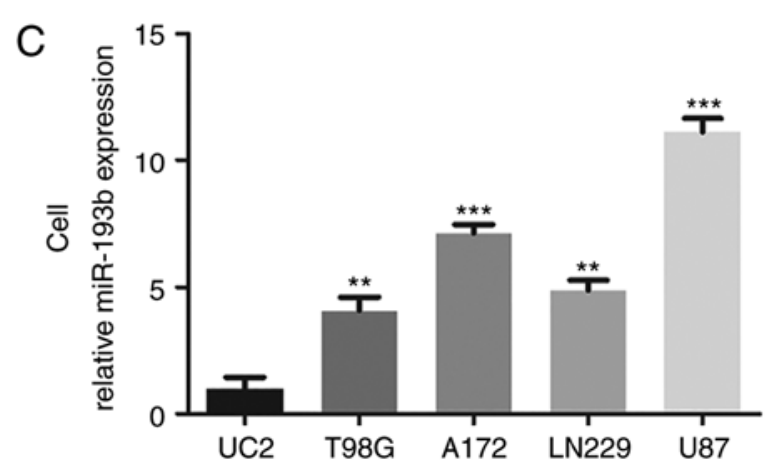

Figure 1. Expression of miR-193b in glioma samples and cell lines. (A) Serum miR-193b levels were higher in patients with glioma than that in healthy individuals. (B) The expression of miR-193b was upregulated in glioma tissues compared with the normal tissues. (C) Increased expression of miR-193b was observed in glioma cell lines compared with normal UC2 cells. ${ }^{* *} \mathrm{P}<0.01$ and ${ }^{* * *} \mathrm{P}<0.001$ vs. corresponding control. miR, microRNA.
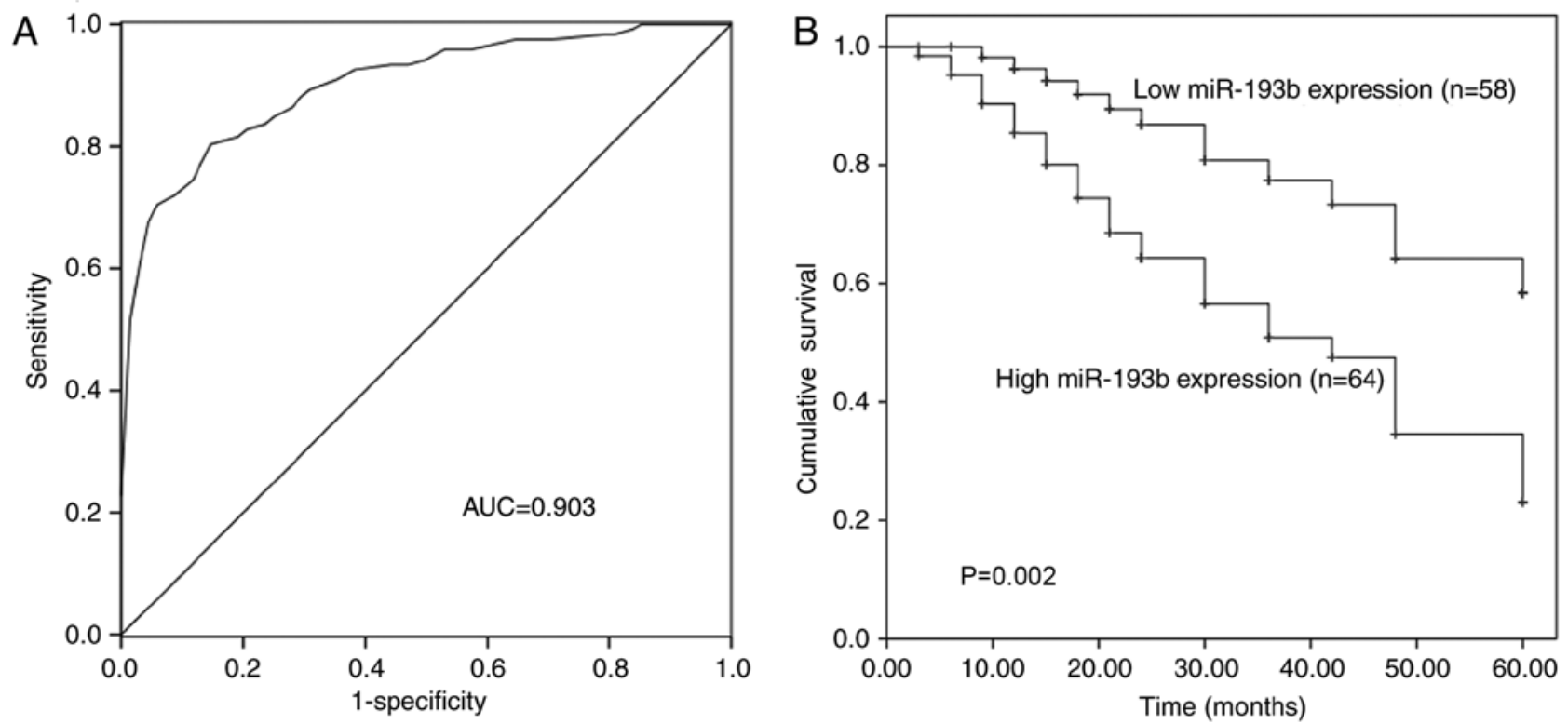

Figure 2. Diagnostic and prognostic significance of miR-193b in glioma. (A) A receiver operating characteristic curve based on miR-193b expression indicated high diagnostic accuracy of miR-193b. (B) Survival curves revealed higher overall survival in patients with low miR-193b expression than in those with high miR-193b expression. miR, microRNA; AUC, area under the curve.

miR-193b expression was evaluated in patients with glioma. The ROC curve was generated, based on the serum levels of miR-193b in the patients (Fig. 2A), and demonstrated that miR-193b had high diagnostic value with an area under the curve of 0.903 . The sensitivity was $79.5 \%$ and the specificity was $86.8 \%$ at the cut-off value of 1.155 , which represented an optimal relative expression value of miR-193b to distinguish glioma patients from healthy individuals.
Kaplan-Meier survival curves were plotted and Cox regression analysis was performed to assess the prognostic value of miR-193b. The expression of miR-193b in glioma tissues was used for these analyses. As shown in Fig. 2B, patients with high expression of miR-193b had low overall survival compared with those with low miR-193b expression $(\mathrm{P}=0.002)$. Furthermore, the univariate and multivariate $\mathrm{Cox}$ regression analysis indicated that miR-193b was associated 
Table II. Cox regression analysis of miR-193b expression and clinicopathological features in patients with glioma.

\begin{tabular}{|c|c|c|c|c|c|c|}
\hline \multirow[b]{2}{*}{ Variable } & \multicolumn{3}{|c|}{ Univariate analysis } & \multicolumn{3}{|c|}{ Multivariate analysis } \\
\hline & HR & $95 \% \mathrm{CI}$ & P-value & HR & $95 \% \mathrm{CI}$ & P-value \\
\hline $\operatorname{miR}-193 b$ & 2.665 & $1.391-5.107$ & 0.003 & 2.877 & $1.410-5.872$ & 0.004 \\
\hline Gender & 1.001 & $0.541-1.853$ & 0.997 & 1.156 & $0.607-2.202$ & 0.660 \\
\hline Age & 1.502 & $0.721-3.126$ & 0.277 & 1.298 & $0.610-2.762$ & 0.498 \\
\hline Tumor size & 1.203 & $0.666-2.173$ & 0.541 & 1.140 & $0.620-2.096$ & 0.672 \\
\hline WHO grade & 2.284 & $1.211-4.138$ & 0.008 & 1.798 & $0.951-3.399$ & 0.071 \\
\hline KPS & 2.166 & $1.199-3.915$ & 0.010 & 1.687 & $0.898-3.171$ & 0.104 \\
\hline
\end{tabular}

miR, microRNA; WHO, World Health Organization; KPS, Karnofsky Performance Scale; HR, hazard ratio; CI, confidence interval.
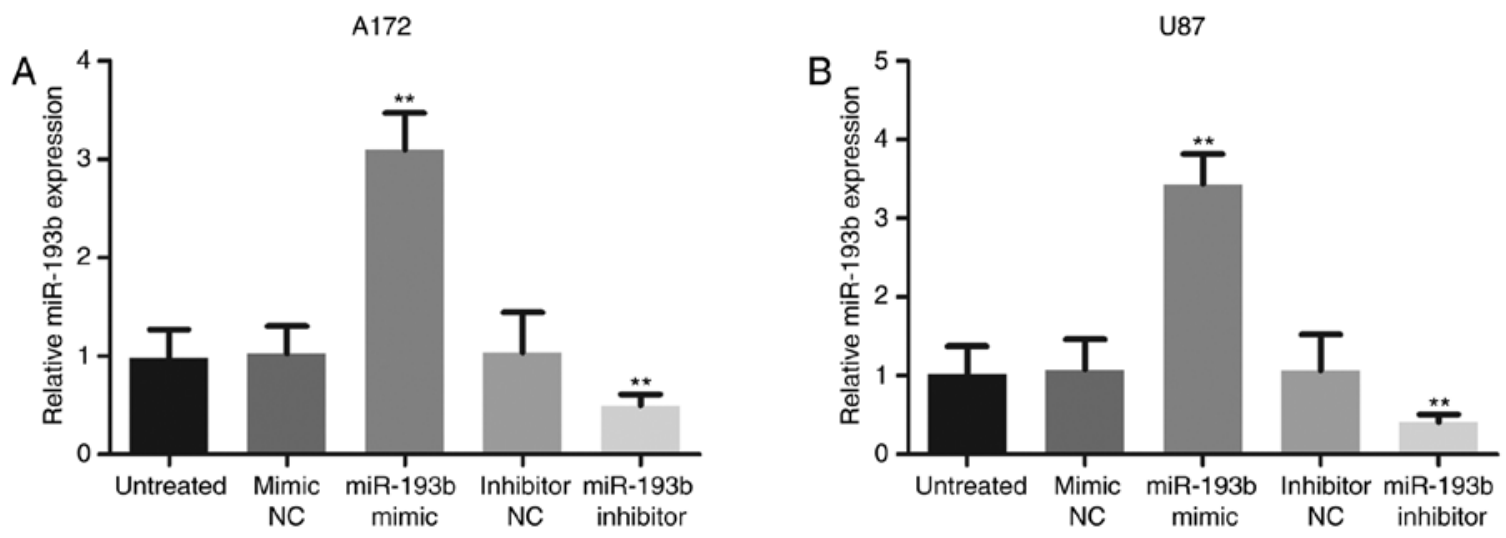

C

A172

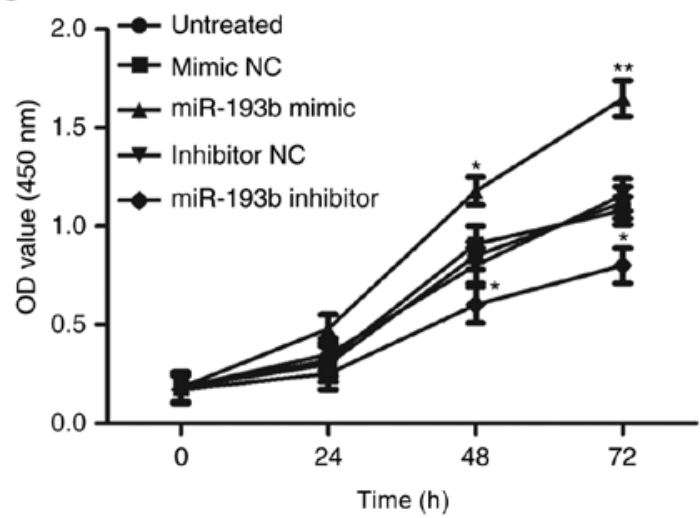

D

U87

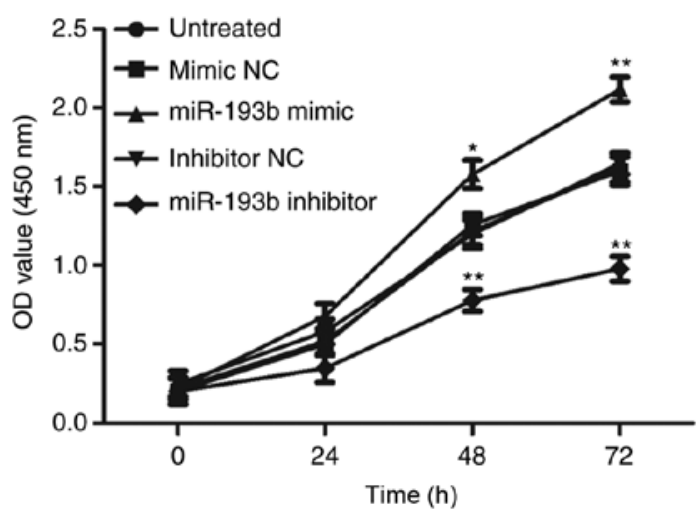

Figure 3. MiR-193b promotes proliferation in glioma cells. Expression of miR-193b was upregulated following transfection with an miR-193b mimic, and downregulated following transfection with an miR-193b inhibitor in glioma (A) A172 and (B) U87 cells. Cell proliferation was promoted by miR-193b overexpression, and suppressed by miR-193b inhibition in (C) A172 and (D) U87 cells. ${ }^{*} \mathrm{P}<0.05$ and ${ }^{* *} \mathrm{P}<0.01$ vs. the untreated group. miR, micro-RNA; NC, negative control; OD, optical density.

with the prognosis of glioma and served as an independent prognostic factor of overall survival in glioma $(\mathrm{P}=0.004$; HR, 2.877; Table II).

Effects of miR-193b on the proliferation of glioma cells. The current study conducted experiments on cells to confirm the functional role of miR-193b in the progression of glioma. Following stable transfection with a mimic or an inhibitor, miR-193b expression was overexpressed or knocked down, respectively, in A172 and U87 cells compared with the corresponding negative controls (all $\mathrm{P}<0.01$; Fig. 3A and $\mathrm{B}$ ). The cell proliferation assay revealed that overexpression of miR-193b contributed to increased glioma cell proliferation, whereas the inhibition of miR-193b expression suppressed the proliferation of the cells (all $\mathrm{P}<0.05$, Fig. $3 \mathrm{C}$ and $\mathrm{D}$ ).

Effects of miR-193b on the migration and invasion of glioma cells. In addition to cell proliferation, the regulatory effects of miR-193b on glioma cell migration and invasion were also analyzed in A172 and U87 cells. Migration and invasion of 

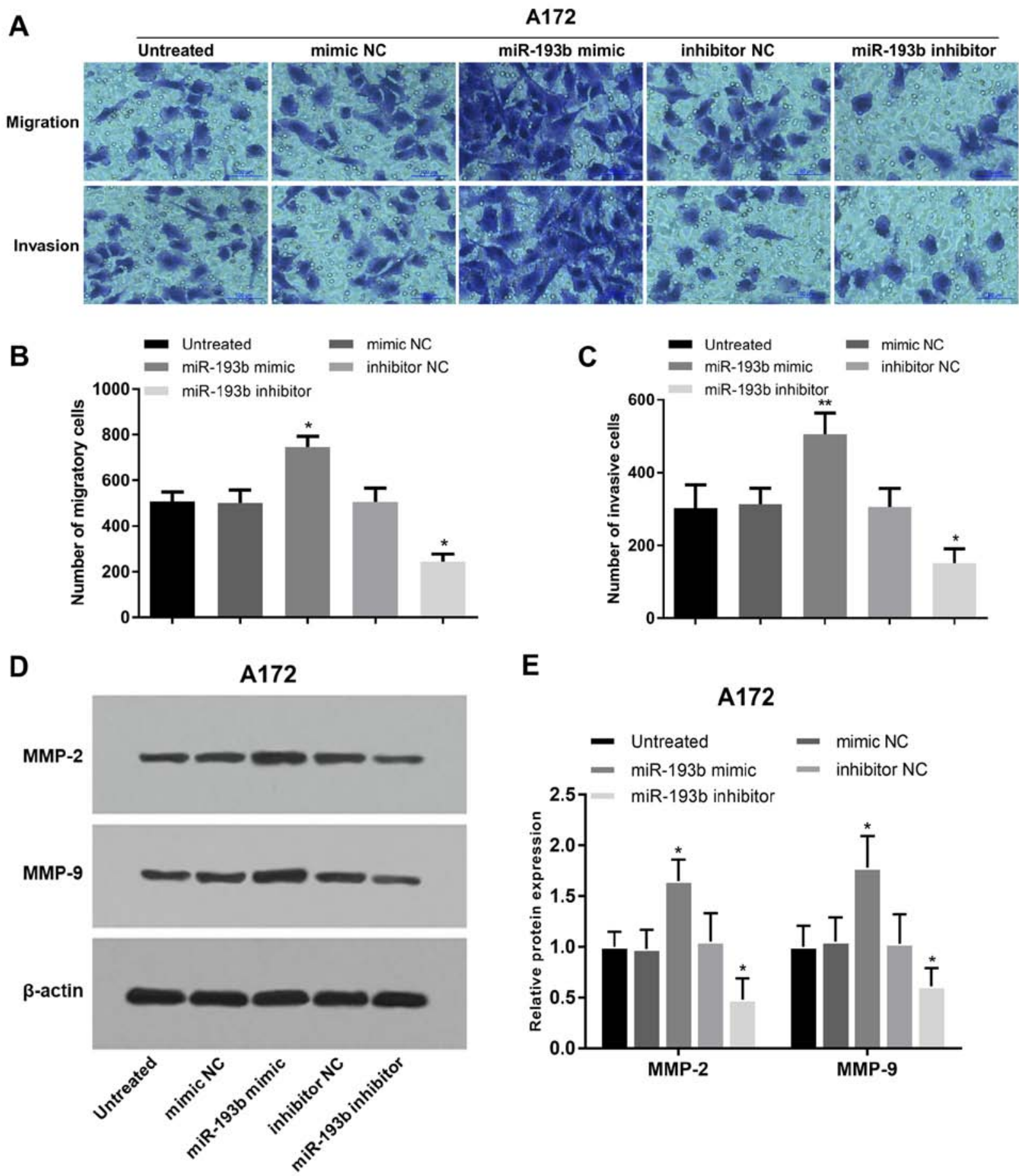

E

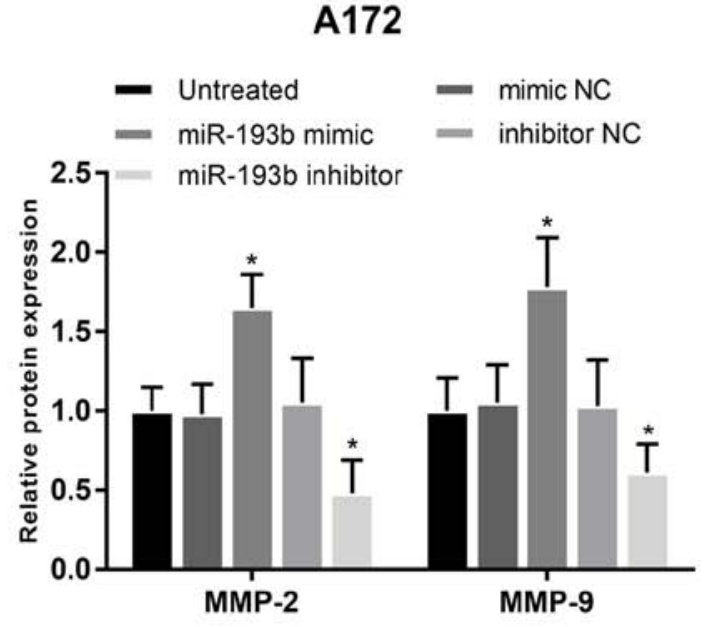

Figure 4. miR-193b leads to enhanced cell migration and invasion of A172 cells. (A-C) Cell migration and invasion of A172 were promoted by the overexpression of miR-193b, and suppressed by its inhibition, following transfection with miR mimic and inhibitor, respectively. (D) Western blot analysis and (E) quantification of MMP-2 and MMP-9 protein expression in A172 cells following transfection. The proteins were increased by the upregulation of miR-193b, and decreased by its inhibition. ${ }^{*} \mathrm{P}<0.05$ and ${ }^{* *} \mathrm{P}<0.01$ vs. the untreated group. miR, microRNA; NC, negative control; MMP, matrix metalloproteinase.

glioma A172 cells were promoted following miR-193b overexpression, whereas they were suppressed following miR-193b inhibition (all $\mathrm{P}<0.05$; Fig. 4A-C). In addition, the proteins involved in cell migration and invasion were examined, and it was found that the protein levels of MMP-2 and MMP-9 were increased following the overexpression of miR-193b and decreased following its inhibition in A172 cells (all $\mathrm{P}<0.05$; Fig. $4 \mathrm{D}$ and $\mathrm{E}$ ). The migration and invasion results were also obtained in another glioma U87 cell line, which exhibited similar regulatory trends on cell migration and invasion (all $\mathrm{P}<0.05$; Fig. 5A-C) and the associated protein levels $(\mathrm{P}<0.05$; Fig. 5D and E) in U87 cells.

\section{Discussion}

Emerging studies have indicated that miRNAs are involved in pivotal events during the pathogenesis of various human diseases (24). Numerous oncogenes and tumor suppressors 
A

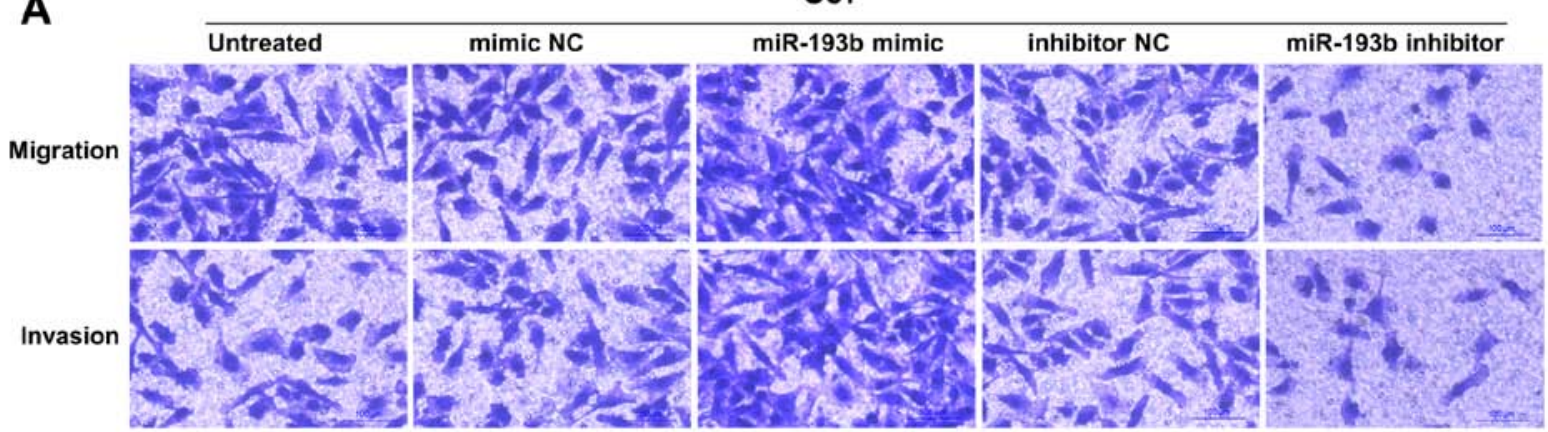

B

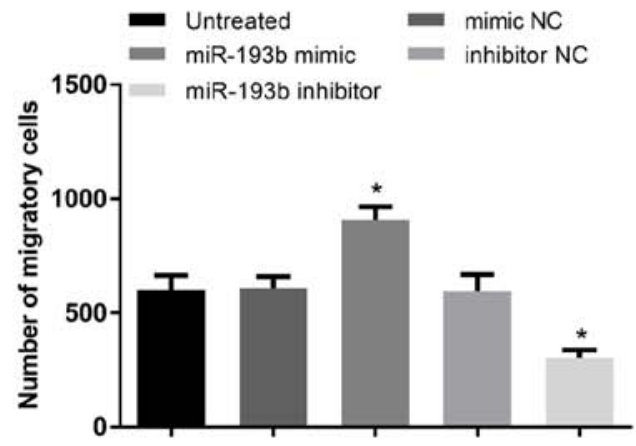

D
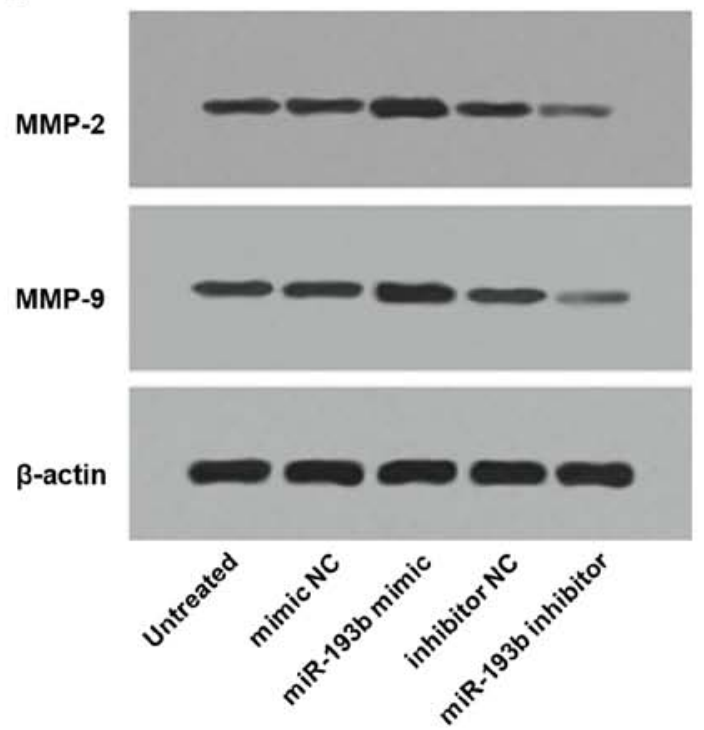

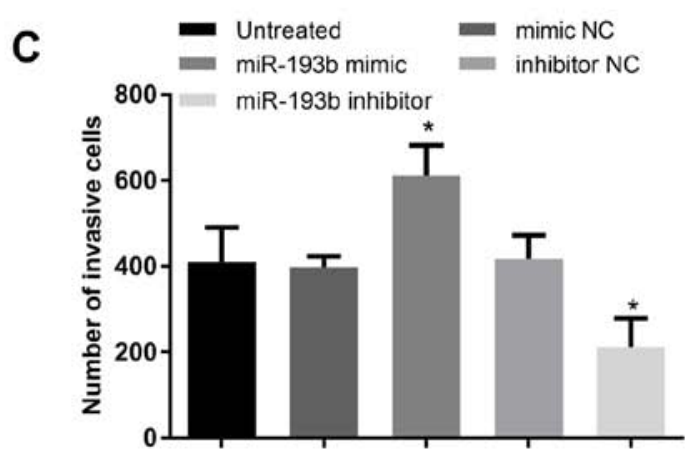

E

U87

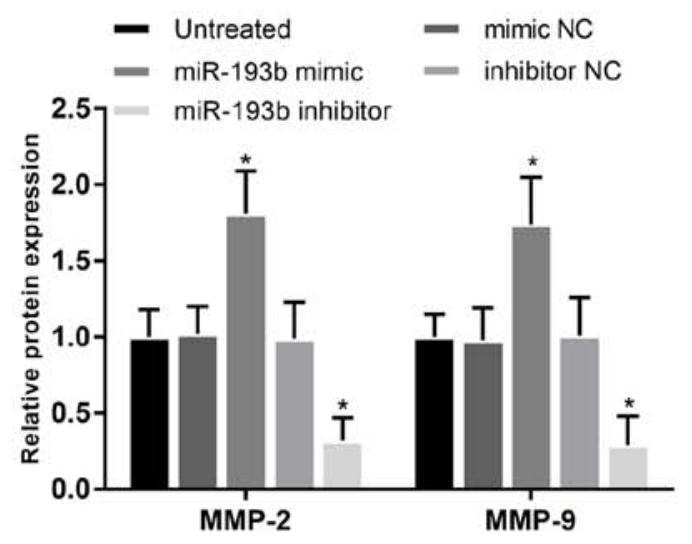

Figure 5. (A-C) Cell migration and invasion of transfected U87 cells. (D) Western blot analysis and (E) quantification of MMP-2 and MMP-9 proteins in U87 cells. " $\mathrm{P}<0.05$ vs. the untreated group. miR, microRNA; NC, negative control; MMP, matrix metalloproteinase.

are regulated by these miRNAs at the post-transcriptional levels (25). The biological function of miRNAs in cancer has received increased attention as a growing number of miRNAs with abnormal expression patterns in tumors have been observed (26). These deregulated miRNAs serve as critical regulators in tumor progression, and can be used as biomarkers for cancer diagnosis and prognosis due to their ectopic expression patterns and stability in serum specimens $(27,28)$. For example, Long et al (29) found that miR-124-3p was decreased in hepatocellular carcinoma samples and predicted prognosis in patients with this malignancy. Another study by Gao et al (14) indicated that serum miR-27a could act as a diagnostic biomarker for the screening of patients with prostate cancer, and demonstrated its effect of promoting tumor cell proliferation. Hence, novel miRNAs, which can accurately screen cancer cases and predict prognosis in patients are required to improve the treatment of malignancies in humans.

Certain miRNAs have been investigated in glioma, and their clinical significance and functional roles were explored in previous studies. For instance, miR-424 expression was downregulated in glioma tissues and cell lines, and participated in the regulation of tumor cell migration, invasion and epithelial-mesenchymal transition (EMT) by targeting the kinesin family member 23 gene (30). The dysregulation of 
miR-129-5p in glioma was demonstrated to suppress glioma cell proliferation and cell cycle by regulating the DNA methyltransferase $3 \alpha$ gene (31). Decreased expression of miR-218-5p was reported in glioma tissues and cells, leading to enhanced cell proliferation, invasion and EMT progression (32). Increased expression of miR-423-3p in glioma predicted poor prognosis, and promoted glioma progression (33). The expression of miR-599 was decreased in glioma tissues compared with normal controls, and was proved to be involved in the progression of glioma (34). In the present study, significantly increased expression of miR-193b was observed in glioma serum, tissues and cell lines. Thus, it is postulated that miR-193b may play a pivotal role in glioma.

Previous studies demonstrated that miR-193b expression was altered in certain types of cancer (17-19). In esophageal squamous cell carcinoma, serum miR-193b expression was downregulated and served as a promising biomarker for the prediction of chemoradiation sensitivity (35). In contrast, the increased expression of miR-193b was detected in colon cancer tissues, and led to increased cell proliferation and decreased cell apoptosis in cells (36). Thus, the expression of miR-193b varies depending on the type of cancer. In glioma, a study reported elevated expression of miR-193b in both glioma tissues and cells (20). Similarly, the present study found upregulated expression of miR-193b in glioma serum samples, tissues and cell lines compared with the corresponding normal controls. Therefore, it is postulated that miR-193b may act as an oncogene in glioma. Additionally, the association between miR-193b expression and clinicopathological characteristics of patients with glioma was further assessed to preliminarily analyze the role of miR-193b in the development of glioma. As expected, the expression of miR-193b was associated with WHO grade and KPS, suggesting that miR-193b may be involved in tumor development.

Given the dysregulation of miR-193b in glioma samples, its clinical significance in glioma diagnosis and prognosis was analyzed. Serum miRNA levels are generally considered as convenient diagnostic tools in cancer (37). Thus, a ROC analysis was performed, based on the serum expression of miR-193b. The results revealed miR-193b as a potential diagnostic biomarker with high sensitivity and specificity. In addition, the prognostic value of miR-193b was evaluated based on the 5-year survival information of the patients with glioma. The Kaplan-Meier survival curves indicated that patients with high miR-193b expression had poor overall survival compared with those with low expression. Furthermore, miR-193b was independently associated with the overall survival, which implied that miR-193b may serve as an independent prognostic biomarker in patients with glioma.

To discover the biological functions of miR-193b in glioma progression, the effects of miR-193b on proliferation, migration and invasion of glioma cells were investigated. The expression of miR-193b was successfully upregulated by an miR-193b mimic, and downregulated by an miR-193b inhibitor. The upregulation of miR-193b was found to promote proliferation, whereas the inhibition of miR-193b in glioma cells had the opposite effect. In addition, similar regulatory effects of miR-193b were observed on the glioma cell migration and invasion, evidenced by the increased migratory and invading cells and the expression of key proteins involved in these processes.
Overall, the findings of the present study suggest that miR-193b may function as an oncogene in the tumor progression of glioma. However, the molecular mechanisms underlying the role of miR-193b in glioma remain unclear. A previous study by Zhong et al (20) indicated that miR-193b promoted glioma cell proliferation by regulating SMAD family member 3 (SMAD3). Thus, we suspected that the effects of miR-193b on cell migration and invasion may also be achieved by targeting SMAD3. Finally, no in vivo experiments were conducted, which is a limitation of the present study. Therefore, further investigations are needed to confirm the role of miR-193b in glioma in vivo and to explore its precise mechanisms.

\section{Acknowledgements}

Not applicable.

\section{Funding}

No funding was received.

\section{Availability of data and materials}

The datasets used and/or analyzed during the present study are available from the corresponding author upon reasonable request.

\section{Authors' contributions}

$\mathrm{MZ}$ and JZ designed the present study and were responsible for writing and revising the manuscript. WZ collected the data and performed the clinical research. HZ performed the cell experiments and data analysis.

\section{Ethics approval and consent to participate}

The present study was approved by the Ethics Committee of Heze Municipal Hospital (approval no. 20070923), and each participant provided written informed consent.

\section{Patient consent for publication}

Not applicable.

\section{Competing interests}

The authors declare that they have no competing interests.

\section{References}

1. Pastuszak Z, Tomczykiewicz K, Piusinska-Macoch R, Stępień A and Kordowska J: The occurrence of tumors of the central nervous system in a clinical observation. Pol Merkur Lekarski 38: 88-92, 2015.

2. Ho VK, Reijneveld JC, Enting RH, Bienfait HP, Robe P, Baumert BG, Visser O and Dutch Society for Neuro-Oncology (LWNO): Changing incidence and improved survival of gliomas. Eur J Cancer 50: 2309-2318, 2014.

3. Malzkorn B and Reifenberger G: Practical implications of integrated glioma classification according to the World Health Organization classification of tumors of the central nervous system 2016. Curr Opin Oncol 28: 494-501, 2016. 
4. Olar A and Aldape KD: Using the molecular classification of glioblastoma to inform personalized treatment. J Pathol 232: 165-177, 2014.

5. Huang L, Liu J, Yu X, Shi L, Liu J, Xiao H and Huang Y: Drug-drug interactions between moxifloxacin and rifampicin based on pharmacokinetics in vivo in rats. Biomed Chromatogr 30: 1591-1598, 2016.

6. Panditharatna E, Yaeger K, Kilburn LB, Packer R and Nazarian J: Clinicopathology of diffuse intrinsic pontine glioma and its redefined genomic and epigenomic landscape. Cancer Genet 208: 367-373, 2015

7. Stoltz K, Sinyuk M, Hale JS, Wu Q, Otvos B, Walker K, Vasanji A, Rich JN, Hjelmeland AB and Lathia JD: Development of a Sox 2 reporter system modeling cellular heterogeneity in glioma. Neuro Oncol 17: 361-371, 2015.

8. Wang H, Song X, Huang Q, Xu T, Yun D, Wang Y, Hu L, Yan Y, Chen H, Lu D and Chen J: LGALS3 promotes treatment resistance in glioblastoma and is associated with tumor risk and prognosis. Cancer Epidemiol Biomarkers Prev 28: 760-769, 2019

9. Zhang L, Zhu Y, Cheng H, Zhang J, Zhu Y, Chen H, Chen L, Qi H, Ren G, Tanget J, et al: The increased expression of estrogen related receptor $\alpha$ correlates with wnt5a and poor prognosis in patients with glioma. Mol Cancer Ther 15, 2018 18: 173-184, 2019.

10. Wang WT and Chen YQ: Circulating miRNAs in cancer: From detection to therapy. J Hematol Oncol 7: 86, 2014.

11. Harrandah AM, Mora RA and Chan EKL: Emerging microRNAs in cancer diagnosis, progression, and immune surveillance. Cancer Lett 438: 126-132, 2018.

12. Chen L, Hu J, Pan L, Yin X, Wang Q and Chen H: Diagnostic and prognostic value of serum miR-99a expression in oral squamous cell carcinoma. Cancer Biomark 23: 333-339, 2018.

13. Deng YH, Deng ZH, Hao H, Wu XL, Gao H, Tang SH and Tang H: MicroRNA-23a promotes colorectal cancer cell survival by targeting PDK4. Exp Cell Res 373: 171-179, 2018.

14. Gao W, Hong Z, Huang H, Zhu A, Lin S, Cheng C, Zhang X, Zou G and Shi Z: miR-27a in serum acts as biomarker for prostate cancer detection and promotes cell proliferation by targeting Sprouty2. Oncol Lett 16: 5291-5298, 2018.

15. Xiang J, Wu Y, Li DS, Wang ZY, Shen Q, Sun TQ, Guan Q and Wang YJ: miR-584 suppresses invasion and cell migration of thyroid carcinoma by regulating the target oncogene ROCK1. Oncol Res Treat 38: 436-440, 2015.

16. Kong Q, Han J, Deng H, Wu F, Guo S and Ye Z: miR-431-5p alters the epithelial-to-mesenchymal transition markers by targeting UROC28 in hepatoma cells. Onco Targets Ther 11: 6489-6503, 2018.

17. Yin W, Nie Y, Chen L, Wang Q, Liu S, He X and Wang W: Deregulation of microRNA-193b affects the proliferation of liver cancer via myeloid cell leukemia-1. Oncol Lett 15: 2781-2788, 2018.

18. Wang L, Zhang Y, Zhao L, Liu S, Yu S, Ma Y and Sun G: MicroRNA-193b inhibits the proliferation, migration and invasion of gastric cancer cells via targeting cyclin D1. Acta Histochem 118: 323-330, 2016

19. Guo F, Luo Y, Mu YF, Qin SL, Qi Y, Qiu YE and Zhong M miR-193b directly targets STMN1 and inhibits the malignant phenotype in colorectal cancer. Am J Cancer Res 6: 2463-2475, 2016.

20. Zhong Q, Wang T, Lu P, Zhang R, Zou J and Yuan S: miR-193b promotes cell proliferation by targeting Smad3 in human glioma. J Neurosci Res 92: 619-626, 2014.

21. Louis DN, Ohgaki H, Wiestler OD, Cavenee WK, Burger PC, Jouvet A, Scheithauer BW and Kleihues P: The 2007 WHO classification of tumours of the central nervous system. Acta Neuropathol 114: 97-109, 2007.
22. Chambless LB, Kistka HM, Parker SL, Hassam-Malani L, McGirt MJ and Thompson RC: The relative value of postoperative versus preoperative Karnofsky Performance Scale scores as a predictor of survival after surgical resection of glioblastoma multiforme. J Neurooncol 121: 359-364, 2015.

23. Livak KJ and Schmittgen TD: Analysis of relative gene expression data using real-time quantitative PCR and the 2(-Delta Delta C(T)) method. Methods 25: 402-408, 2001.

24. Di Leva G, Garofalo M and Croce CM: MicroRNAs in cancer Annu Rev Pathol 9: 287-314, 2014

25. Fan Z, Cui H, Xu X, Lin Z, Zhang X, Kang L, Han B, Meng J, Yan Z, Yan X and Jiao S: MiR-125a suppresses tumor growth, invasion and metastasis in cervical cancer by targeting STAT3. Oncotarget 6: 25266-25280, 2015.

26. Dassow H and Aigner A: MicroRNAs (miRNAs) in colorectal cancer: From aberrant expression towards therapy. Curr Pharm Des 19: 1242-1252, 2013.

27. Bertoli G, Cava C and Castiglioni I: MicroRNAs: New biomarkers for diagnosis, prognosis, therapy prediction and therapeutic tools for breast cancer. Theranostics 5: 1122-1143, 2015.

28. Guo SJ, Zeng HX, Huang P, Wang S, Xie CH and Li SJ MiR-508-3p inhibits cell invasion and epithelial-mesenchymal transition by targeting ZEB1 in triple-negative breast cancer. Eur Rev Med Pharmacol Sci 22: 6379-6385, 2018

29. Long HD, Ma YS, Yang HQ, Xue SB, Liu JB, Yu F, Lv ZW, Li JY, Xie RT, Chang ZY, et al: Reduced hsa-miR-124-3p levels are associated with the poor survival of patients with hepatocellular carcinoma. Mol Biol Rep 45: 2615-2623, 2018.

30. Zhao C, Wang XB, Zhang YH, Zhou YM, Yin Q and Yao WC: MicroRNA-424 inhibits cell migration, invasion and epithelial-mesenchymal transition in human glioma by targeting KIF23 and functions as a novel prognostic predictor. Eur Rev Med Pharmacol Sci 22: 6369-6378, 2018.

31. Gu X, Gong H, Shen L and Gu Q: MicroRNA-129-5p inhibits human glioma cell proliferation and induces cell cycle arrest by directly targeting DNMT3A. Am J Transl Res 10: 2834-2847, 2018.

32. Li Z, Qian R, Zhang J and Shi X: MiR-218-5p targets LHFPL3 to regulate proliferation, migration and epithelial-mesenchymal transitions of human glioma cells. Biosci Rep 39: pii: BSR20180879, 2019

33. Xu J, He J, Huang H, Peng R and Xi J: MicroRNA-423-3p promotes glioma growth by targeting PANX2. Oncol Lett 16: 179-188, 2018

34. Jiang Y, Wang X, Zhang J and Lai R: MicroRNA-599 suppresses glioma progression by targeting RAB27B. Oncol Lett 16 : 1243-1252, 2018.

35. Chan CM, Lai KKY, Ng EKO, Kiang MN, Kwok TWH, Wang HK, Chan KW, Law TT, Tong DK, Chan KT, et al: Serum microRNA-193b as a promising biomarker for prediction of chemoradiation sensitivity in esophageal squamous cell carcinoma patients. Oncol Lett 15: 3273-3280, 2018

36. Wu K, Zhao Z, Ma J, Chen J, Peng J, Yang S and He Y: Deregulation of miR-193b affects the growth of colon cancer cells via transforming growth factor $\beta$ and regulation of the SMAD3 pathway. Oncol Lett 13: 2557-2562, 2017

37. $\mathrm{Lu} \mathrm{X}$ and $\mathrm{Lu} \mathrm{J}$ : The significance of detection of serum miR-423-5p and miR-484 for diagnosis of colorectal cancer. Clin Lab 61: 187-190, 2015.

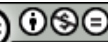

This work is licensed under a Creative Commons Attribution-NonCommercial-NoDerivatives 4.0 International (CC BY-NC-ND 4.0) License. 